Alfredo M. Oliveira Filho ${ }^{1}$

Marli T. V. Melo ${ }^{1}$

Celso E. Santos 1

Oswaldo F. Faria Filho 2

Francisco C. F. Carneiro ${ }^{2}$

José Wellington Oliveira-Lima 2

João Batista Furtado Vieira 2

Francisco Vieira Gadelha 2

Jorge Ishihata 3

\section{Tratamentos focais e totais com inseticidas de ação residual para o controle de Triatoma brasiliensis e Triatoma pseudomaculata no Nordeste brasileiro}

\author{
Focal and total residual insecticide spraying \\ to control Triatoma brasiliensis and Triatoma \\ pseudomaculata in Northeast Brazil
}

\footnotetext{
1 Núcleo de Pesquisas de Produtos Naturais, Centro de Ciências da Saúde, Universidade Federal do Rio de Janeiro. Av. Brigadeiro Trompowski $s / n o$, Rio de Janeiro, $R J$ 21941-590, Brasil.

2 Fundação Nacional de Saúde.

Av. Santos Dumont 1856, Fortaleza, $C E$

60150-160, Brasil.

3 Fundação Nacional de Saúde.

Setor de Autarquias Sul, Q4 Bloco N, Brasília, DF 70070-500, Brasil.
}

\begin{abstract}
To efficiently control the triatomines Triatoma brasiliensis and Triatoma pseudomaculata, a field trial was performed to compare conventional versus focal spraying of deltamethrin 5\% SC at $25 \mathrm{mg}$ a.i./ $\mathrm{m}^{2}$ and the slow-release organophosphate malathion 8.3\% SR at $2 \mathrm{~g}$ a.i./ $\mathrm{m}^{2}$. The assay took place in the county of Boa Viagem, Ceará State, with 1541 households, randomly separated into 4 groups. Two of them received focal spraying: PT, treated with deltamethrin indoors and in the peridomicile, and PL, which received slow-release malathion in the same circumstances. The other groups received conventional, i.e., total application: PT with deltamethrin in the intra- and peridomicile, and PL, which was treated with deltamethrin indoors and slow-release malathion in the peridomicile. Entomological surveys at 6 and 12 months post-treatment showed better results for mixed treatment, the PL group, probably due to good indoor performance for the pyrethroid and better performance of the slow-release formulation under the hostile peridomiciliary conditions. Domestic animal shelters underwent numerous modifications over the course of the year, fostering reduced insecticide performance in the peridomicile.
\end{abstract}

Key words Chagas Disease; Triatominae; Insect Vectors; Vector Control; Prevention and Control

Resumo Para controle eficiente dos triatomíneos Triatoma brasiliensis $e$ Triatoma pseudomaculata, foi feito um ensaio de campo em Boa Viagem, Ceará, de modo a comparar a borrifação convencional versus tratamento focal com deltametrina 5\% SC, dose $25 \mathrm{mg}$ i.a. $/ \mathrm{m}^{2}$ e o organofosforado malation lenta liberação $8.3 \%$ SR, dose $2 \mathrm{~g}$ i.a. $/ \mathrm{m}^{2}$. O ensaio incluiu aleatoriamente 1.541 casas, separadas em quatro grupos. Em dois deles foi aplicada borrifação focal - tratamento PT com deltametrina dentro das casas e no peridomicílio e PL que recebeu malation lenta liberação nas mesmas circunstâncias. Os outros dois tiveram tratamento convencional, isto é, aplicação total - PT com deltametrina no intra e peridomicílio e PL, tratado com deltametrina dentro das casas e malation lenta liberação no peridomicílio. As avaliações entomológicas aos 6 e 12 meses pós-tratamentos mostraram melhor resultado para o tratamento misto, grupo PL, provavelmente em decorrência da boa performance do piretróide dentro das casas e da formulação de lenta liberação nas condições hostis do peridomicílio. Os abrigos dos animais domésticos sofreram modificações ao longo do ano, colaborando com a redução da performance dos inseticidas no peridomicílio.

Palavras-chave Doença de Chagas; Triatominae; Insetos Vetores; Controle de Vetores; Prevenção e Controle 


\section{Introdução}

Os inseticidas usados nas campanhas de controle dos vetores da doença de Chagas apresentam, em geral, pouco efeito residual, uma vez que as doses recomendadas produzem alto impacto inicial nas populações de triatomíneos, mas as doses letais não se mantêm nas superfícies tratadas mais do que alguns meses (Oliveira Filho, 1983; Pinchin et al., 1984; Oliveira Filho \& Melo, 1987). Isto se faz notar mais claramente nos tratamentos realizados no peridomicílio, mesmo quando se trata do uso de piretróides sintéticos de última geração (Pinchin et al., 1980, 1981b; Oliveira Filho et al., 1988a, 1988b). Este fato é esperado, pois as formulações convencionais mais usadas atualmente são os pós molháveis e as suspensões concentradas, que representam somente um veículo para os princípios ativos e não fornecem proteção contra o ar, luz, radiação ultravioleta e umidade, nem contra fatores físicos, tais como chuva, vento e outros agentes, inclusive seres humanos e animais domésticos, os quais promovem a erosão das superfícies tratadas removendo o inseticida aí depositado.

Como conseqüência do exposto, aplicações repetidas dos inseticidas fazem-se necessárias, em especial quando os triatomíneos presentes podem viver também nas áreas silvestres ao redor das habitações humanas, de onde invadem constantemente o peridomicílio e, daí, as casas. No Brasil, esta situação, tida como de alta pressão de reinfestação, é observada principalmente nas áreas da região nordeste, nas quais se registra a presença de Triatoma brasiliensis e Triatoma pseudomaculata.

Com o intuito de aumentar o efeito residual de inseticidas usados no tratamento de paredes, tetos e construções peridomiciliares - inclusive aquelas que não são protegidas por qualquer tipo de teto, como poleiros, currais, montes de lenha e de materiais de construção - o Laboratório de Biologia do Núcleo de Pesquisas de Produtos Naturais da Universidade Federal do Rio de Janeiro (NPPN) desenvolveu uma formulação de lenta liberação à base de acetato de polivinila e copolímeros. Ensaios de laboratório e de campo efetuados anteriormente demonstraram sua capacidade de permanecer em atividade por 18 a 24 meses após tratamento único, mesmo quando eram usados os organofosforados malation ou fenitrotion, que apresentam curta persistência em aplicações residuais convencionais (Pinchin et al., 1979; Oliveira Filho, 1988, 1995, 1997).

Neste ensaio de campo de larga escala, buscamos comparar o efeito inicial e residual de uma formulação de lenta liberação de malation com uma formulação convencional do piretróide deltametrina e aplicações combinadas de ambos, bem como aplicações focais de cada um. Ele foi realizado em área considerada de alta pressão de reinfestação por estarem infestadas por T. brasiliensis e T. pseudomaculata, apresentando também alta dispersão destes triatomíneos e quase $25 \%$ das unidades domiciliares infestadas. Outra característica importante e típica deste tipo de aplicação é que ele era preponderante no peridomicílio, quando comparado ao intradomicílio. Todas as operações de campo foram executadas em estreita colaboração entre o NPPN e a Fundação Nacional de Saúde (FNS/CE).

\section{Materiais e métodos}

A área do ensaio estava toda contida no Município de Boa Viagem, Estado do Ceará, e compreendia 68 localidades, em que predominam aglomerados de casas rurais (sítios, fazendas e povoados), somando-se 1.541 casas tratadas. Os inseticidas utilizados foram o malation lenta liberação (Duration M, LL 8,3\% - Iharabras), aplicado na dose de $2 \mathrm{~g}$ i.a. $/ \mathrm{m}^{2}$, e a deltametrina suspensão concentrada ou flowable (5\% SC - Quimio), aplicada a 25 mg i.a. $/ \mathrm{m}^{2}$.

As localidades selecionadas para o ensaio foram alocadas aleatoriamente em quatro grupos. Em todos eles, o tratamento foi feito utilizando-se bombas Hudson-X-Pert, com capacidade para dez litros, munidas de bicos Teejet 8002 , dispersando $760 \mathrm{ml} / \mathrm{min}$, à pressão de 50 psi. Nestas condições, a $45 \mathrm{~cm}$ de distância da superfície, conseguia-se uma faixa tratada de $75 \mathrm{~cm}$ de largura. Antes da aplicação, toda a comida, água potável, utensílios de cozinha e animais domésticos foram removidos. Os móveis foram afastados da parede e os cartazes removidos.

Foram executados dois grandes tipos de tratamento. O total ou tradicional, em que todas as paredes internas - parte inferior do teto, parte inferior e posterior dos móveis - e, por fora, os beirais do telhado foram tratados. Neste caso, o peridomicílio era todo tratado, em particular, os abrigos cobertos de animais. $\mathrm{O}$ segundo tipo foi chamado focal por concentrar-se principalmente ao redor das fontes alimentares dos triatomíneos, isto é, das possíveis fontes de sangue. Nesta modalidade não se borrifaram paredes lisas, a não ser que houvesse fonte de sangue próxima, quando então se borrifava ao seu redor. Foram também tratados os beirais e os frechais do teto, camas, ar- 
madores e cordas para pendurar redes de dormir, as partes posteriores de quadros e outros objetos nas paredes. Somente se borrifavam as paredes de mal acabamento dos quartos de dormir ou de outro local usado para este fim. Móveis, caixas, montes de lenha, telhas e tijolos foram tratados quando estavam a até dois metros da fonte alimentar dos triatomíneos. No peridomicílio foram borrifados abrigos de animais, incluindo beirais e frechais, mas as paredes lisas não eram tratadas. Também receberam inseticida os poleiros, chiqueiros e currais próximos de locais onde dormiam ou descansavam os animais. Pilhas de tijolos, telhas e lenha somente eram tratadas, no peridomicílio, quando apresentavam vestígios da presença de triatomíneos.

Dos quatro grupos de tratamento, dois receberam aplicação focal - o grupo PF, tratado com o piretróide deltametrina, e o LF, tratado com o malation lenta liberação em ambos, no intra e no peridomicílio. Os outros dois receberam aplicação total - o PT com piretróide no intra e peridomicílio, e o PL com piretróide no intra e malation lenta liberação no peridomicílio. Buscava-se, com isto, comparar as performances do piretróide convencional com a do organofosforado em formulação de lenta liberação, visando à redução da freqüência dos tratamentos, da mesma forma que comparar o tratamento total com o focal, com o objetivo de economizar inseticida nos tratamentos. O grupo misto piretróide no intra e LL no peridomicílio foi feito para evitar o tratamento total de LL no intradomicílio, por ser inapropriado para este fim, já que formava um filme plástico de difícil remoção sobre o piso respingado (Pin- chin et al., 1981a; Oliveira Filho, 1995, 1996;

Briceño-León et al., 1997).

Antes da borrifação efetuou-se uma leitura pré tratamento, buscando-se ativamente a presença de triatomíneos das duas espécies no intra e peridomicílio com o auxílio de um desalojante. As leituras pós-tratamento foram realizadas aos 6 e 12 meses depois da borrifação feita entre setembro e novembro de 1996. Em todas as leituras, a presença de 1 triatomíneo vivo - nos estágios de ovo, ninfa ou adulto - era suficiente para se considerar o local infestado.

\section{Resultados}

Os dados obtidos nas leituras pré-tratamento e até 1 ano pós-tratamento, por grupo tratado, separando-se os resultados obtidos na infestação do intra, peridomicílio e unidade domiciliar, estão expressos na Tabela 1. Com relação às unidades domiciliares observa-se que as percentagens de infestação variaram de 20,7 a $25,4 \%$ no pré-tratamento, caindo para 5,1 a $8,5 \%$ na leitura de 6 meses, aumentando um pouco - entre 6,8 e $16,1 \%$ - na leitura de 12 meses. Nesta última leitura observa-se separação das performances entre os grupos. O melhor deles foi o PL com 6,8\% de infestação, seguido do PT e PF, estes quase iguais, respectivamente com 11,2 e 11,9\%. Por último aparece o LF, já com $16,1 \%$ de unidades infestadas por qualquer das duas espécies presentes na área.

Observa-se também que as infestações no peridomicílio sempre apresentaram valores significativamente maiores que os do intradomi-

Tabela 1

Percentagens e, entre parênteses, números de casas infestadas por Triatoma brasiliensis ou Triatoma pseudomaculata no intradomicílio, no peridomicílio e na unidade domiciliar, nas leituras pré-tratamento e de 6 e 12 meses pós-tratamento, por grupo tratado e no total dos quatro grupos, no ensaio de Boa Viagem, Ceará.

\begin{tabular}{|c|c|c|c|c|c|c|c|c|c|c|c|c|}
\hline \multirow[t]{2}{*}{ Tratamento } & \multicolumn{4}{|c|}{ Pré-tratamento } & \multicolumn{4}{|c|}{6 meses pós-tratamento } & \multicolumn{4}{|c|}{12 meses pós-tratamento } \\
\hline & $\mathrm{n}$ UDs & Intra & Peri & UDs & $\mathrm{n}$ UDs & Intra & Peri & UDs & $\mathrm{n}$ UDs & Intra & Peri & UDs \\
\hline Lenta Focal & 359 & $4,7(17)$ & $22,0(79)$ & $25,3(91)$ & 353 & $2,8(10)$ & $5,9(21)$ & $8,5(30)$ & 348 & $4,6(16)$ & $12,1(42)$ & $16,1(56)$ \\
\hline Piret. Focal & 338 & $3,3(11)$ & $23,4(79)$ & $25,4(86)$ & 334 & $0,6(2)$ & $4,5(15)$ & $5,1(17)$ & 327 & $1,2(4)$ & $10,7(35)$ & 11,9 (39) \\
\hline Piret. Lenta & 429 & $5,1(22)$ & $17,2(74)$ & 20,7 (89) & 420 & $0,7(3)$ & $5,7(24)$ & $6,0(25)$ & 410 & $1,0(4)$ & $5,9(24)$ & $6,8(28)$ \\
\hline Piret. Total & 415 & $6,7(28)$ & $19,0(79)$ & $23,4(97)$ & 412 & $0,5(2)$ & $6,8(28)$ & $7,0(29)$ & 402 & $1,5(6)$ & $10,0(40)$ & $11,2(45)$ \\
\hline Todos & 1541 & $5,1(78)$ & $20,2(311)$ & $23,6(363)$ & 1519 & $1,1(17)$ & $5,8(88)$ & $6,6(101)$ & 1487 & $2,0(30)$ & $9,5(141)$ & $11,3(168)$ \\
\hline
\end{tabular}

UDs = unidades domiciliares; Intra = intradomicílio;

Peri = peridomicílio; Lenta Focal = malation lenta liberação focal intra e peridomicílio .

Piret. Focal $=$ piretróide deltametrina focal intra e peridomicílio.

Piret. Lenta $=$ piretróide total no intra e lenta liberação total no peridomicílio.

Piret. Total $=$ piretróide total intra e peridomicílio. 
cílio. Tanto nas leituras pré-tratamento como nas pós-tratamento, o peridomicílio contribuiu sempre com mais de $80 \%$ das infestações, principalmente quando considerado o conjunto dos dados sem particularizar os tratamentos, como se vê na Figura 1.

Com referência às infestações no intra e no peridomicílio observa-se que houve queda significativa da infestação em todos os grupos nas leituras de 6 e 12 meses em relação àquela realizada antes do tratamento. Aos 12 meses notase, para o intradomicílio, que não há diferenças entre os grupos PF, PL e PT (de 1,0 a 1,5\% de infestação), mas sim com relação ao grupo LF que apresentou a pior performance $-4,6 \%$ de infestação -, praticamente a mesma do prétratamento. Já para o peridomicílio, aos 12 meses somente se destaca o grupo PL com a menor taxa de infestação - 5,9\% -, quando comparado aos outros três, que variavam de 10 a $12,1 \%$. Estes dados podem ser melhor comparados na Figura 2.

Se, no entanto, levarmos em conta apenas a redução das percentagens de infestação entre o pré-tratamento e a leitura de um ano póstratamento - como, por exemplo, nos grupos piretróide total e piretróide intra/LL peri - veremos que foram semelhantes tanto no intra $(\mathrm{p}=0,274)$ quanto no peridomicílio $(\mathrm{p}=0,245)$ (Faria Filho et al., 1998).

Quando se fala em domicílios infestados nas leituras pós-tratamento, em geral se imagi- na que o tratamento falhou na eliminação dos triatomíneos presentes nas casas anteriormente positivas. Nossos dados mostraram que, nas circunstâncias estudadas, apenas parte das casas se mantiveram positivas. Cerca de metade das positivas, tanto na leitura de 6 quanto na de 12 meses, foram tidas como negativas no pré-tratamento (Figura 3). Pode-se então levantar duas hipóteses para explicar isto - existiu, de fato, infestação posterior ao tratamento ou houve falha no levantamento triatomínico feito com a busca ativa destes insetos. Uma análise pormenorizada dos dados, casa a casa, fazse necessária para responder a esta questão.

\section{Conclusões}

Os resultados obtidos indicam que:

- O grupo que recebeu piretróide no intradomicílio e lenta-liberação no peridomicílio obteve, de forma consistente, os melhores resultados, indicando que uma aplicação mista evitaria a necessidade de tratamentos freqüentes. Este resultado era esperado, até certo ponto, já que o piretróide causa alto impacto inicial e preserva sua atividade por alguns meses, neste ambiente, por estar protegido no interior da casa, enquanto o inseticida de lenta-liberação resiste mais às condições hostis do peridomicílio. - Não houve grande diferença entre os grupos de domicílios tratados com piretróide total

Figura 1

Percentagens de intradomicílios, peridomicílios e unidades domiciliares infestadas por triatomíneos nas leituras pré-tratamento e de 6 e 12 meses pós-tratamento no total dos quatro grupos tratados no ensaio de Boa Viagem, Ceará.

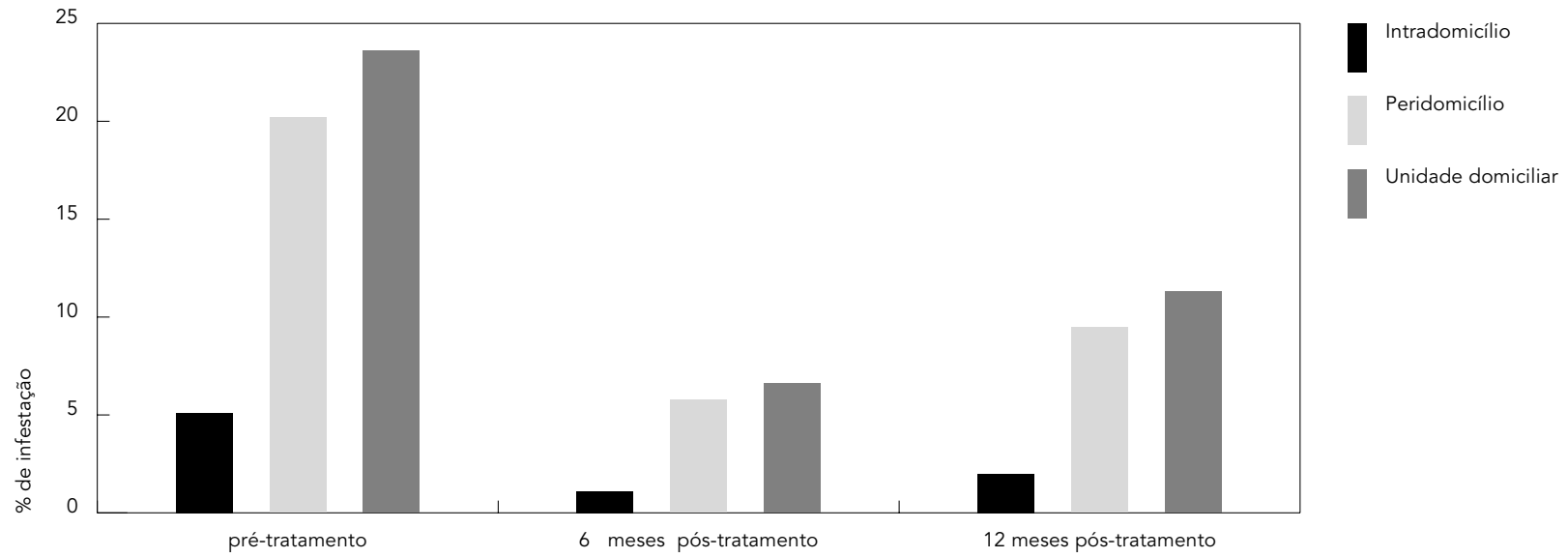




\section{Figura 2}

Percentagens de unidades domiciliares, intra e peridomicílios infestados por Triatoma brasiliensis ou Triatoma pseudomaculata nas leituras pré e de 6 e 12 meses pós-tratamento, por grupo tratado, no ensaio de Boa Viagem, Ceará.

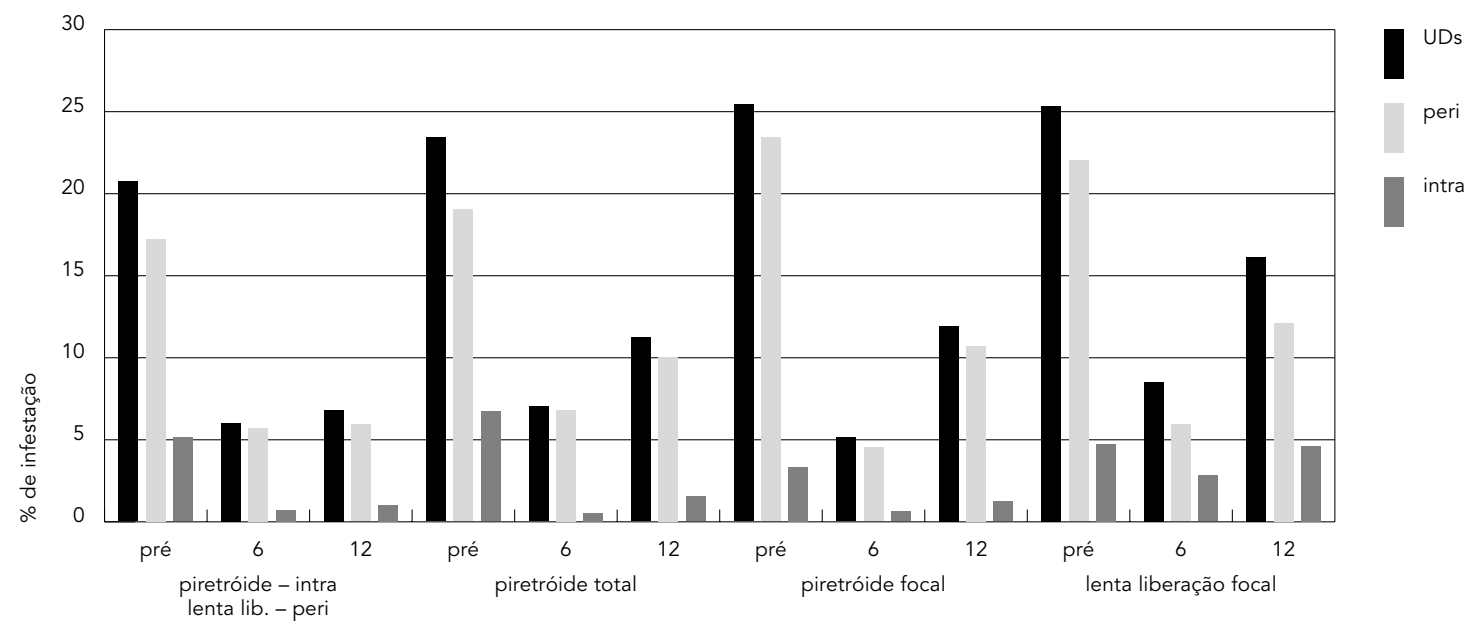

UDs $=$ unidades domiciliares; Peri $=$ peridomicílio; Intra $=$ Intradomicílio

Lenta liberação = malation $8,3 \%$ - dose $2 \mathrm{~g}$ i.a. $/ \mathrm{m}^{2}$; Piretróide = deltametrina $5 \%$ SC - dose $25 \mathrm{mg}$ i.a. $/ \mathrm{m}^{2}$.

Figura 3

Percentagens de unidades domiciliares infestadas com triatomíneos nas leituras pré- 6 e 12 meses pós tratamento; unidades infestadas no pré-tratamento que se mantiveram positivas pós-tratamento (posit. pré/posit pós)

e unidades que eram negativas no pré-tratamento e que se infestaram posteriormente (negat. pré/posit. pós), no ensaio de Boa Viagem, Ceará.

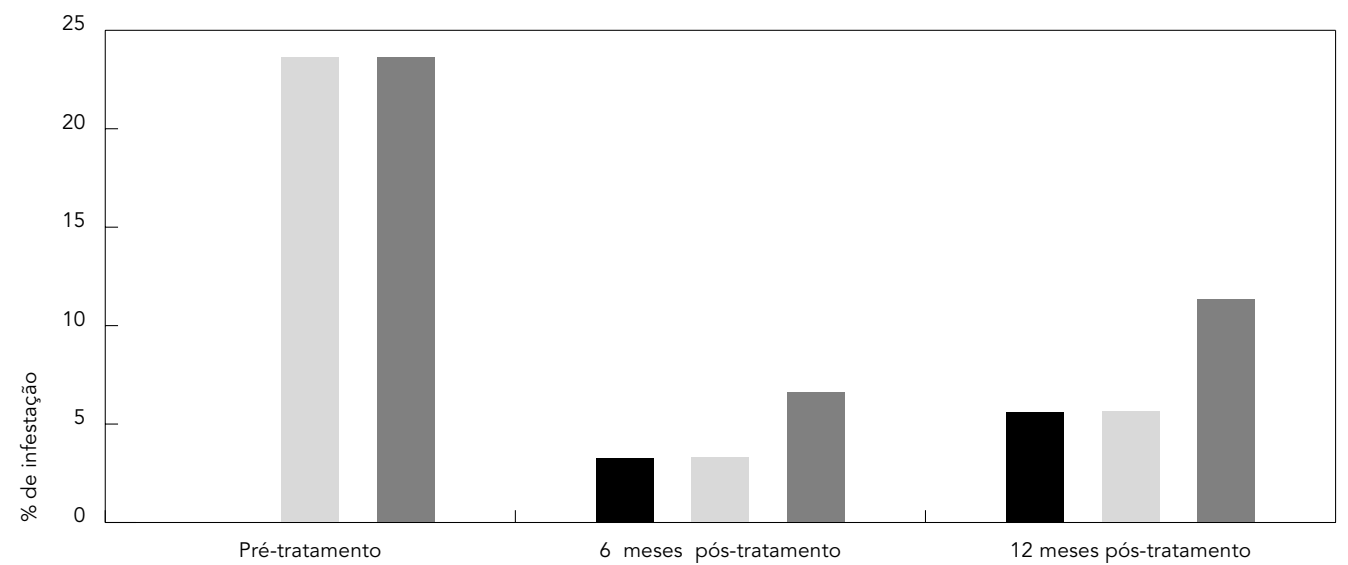

Negat. pré/posit. pós

Posit. pré/mantidas posit.

Posit. pré/posit. pós 
e com focal. Este último tipo de aplicação poderia, então, ser empregado nas campanhas de controle com performance parecida à das aplicações tradicionais recomendadas pela Organização Mundial da Saúde e praticadas historicamente pela FNS.

- A aplicação da formulação de lenta liberação no interior das casas só é aceitável, para os moradores, quando feita de maneira focal. Mesmo assim, o mais recomendável seria a aplicação de formulação convencional no intradomicílio pela facilidade de limpeza dos locais não alvo tratados, em especial, o piso das casas, respingado pelo tratamento das paredes e teto.

- O peridomicílio é o principal fator de infestação das unidades domiciliares nas áreas infestadas por T. brasiliensis e T. pseudomacula$t a$, respondendo por cerca de $80 \%$ dos casos. Resta avaliar se vale a pena efetuar o seu tratamento com a freqüência necessária para mantê-lo com baixa infestação, já que, mesmo sem tratamento, o peridomicílio pode representar uma barreira à entrada destes triatomíneos no interior da casa.

- O peridomicílio sofre modificações muito freqüentes com o desmantelamento e reconstrução dos abrigos temporários de animais domésticos e a descaracterização dos locais trata-

\section{Agradecimentos}

Os autores agradecem a importante colaboração de todos os integrantes do Laboratório de Biologia do Núcleo de Pesquisas de Produtos Naturais, Elizabete G. da Costa, Elza L. B. Lustosa, Aline T. M. Oliveira, Orbino Cosme Damião, Joaquim José de Souza e da Fundação Nacional de Saúde - FNS/Fortaleza, assim como a todos os motoristas, inspetores e guardas da FNS/Ceará que participaram deste ensaio. Recursos financeiros foram obtidos da FNS, World Health Organization/United Nations Development Program/ World Bank/Transmitted Diseases Research, Conselho de Ensino para Graduados, Universidade Federal do Rio de Janeiro e Conselho Nacional de Desenvolvimento Científico e Tecnológico. dos com a introdução de novos materiais não tratados, principalmente paus tirados do ambiente silvestre que já podem trazer consigo novos exemplares de triatomíneos. Deste modo, o peridomicílio, 12 meses pós-tratamento, pode ser completamente distinto daquele que existia à época do tratamento, não se podendo atribuir sempre, ao inseticida ou à operação, a falha em manter o ambiente não infestado.

- Quando se pensa no controle da transmissão da doença de Chagas em regiões com características biológicas e ecológicas das espécies mencionadas, deve-se ter em mente que seria mais fácil controlar apenas as infestações intradomiciliares, com custo significativamente menor, e ainda assim impedir significativamente o contato homem-inseto. Nesta opção poder-se-ia tratar somente o interior da casa e os abrigos de animais muito próximos à mesma, além de convencer seus moradores a afastá-los daí e a não mantê-los dentro de casa. Deste modo, o interior das habitações humanas poderia permanecer livre de infestação com o tratamento anual de apenas pequena parcela de cerca de $5 \%$ das mesmas. As vantagens são óbvias quanto ao custo/eficiência (Oliveira Filho, 1989) e quanto à menor contaminação ambiental (Oliveira Filho, 1998).

\section{Referências}

BRICEÑO-LEON, R.; OLIVEIRA FILHO, A. M.; PAULONE, I. \& SEGURA, E., 1997. Triatomine bugs. In: Vector Control-Methods for Use by Individuals and Communities (J. A. Rozendaal, ed.), pp. 210-236, Geneva: World Health Organization.

FARIA FILHO, O. F.; GADELHA, F. V.; CARNEIRO, F. C. F; LIMA, J. N. O. \& OLIVEIRA FILHO, A. M., 1998. Ensaio para avaliação do efeito residual do Duration M no controle do Triatoma brasiliensis e $T$. pseudomaculata. Revista da Sociedade Brasileira de Medicina Tropical, 31 (Sup. 1):58.

OLIVEIRA FILHO, A. M., 1983. Avaliação do inseticida chlorphoxim (OMS 1197) contra triatomíneos Ensaio de campo. Ciência e Cultura, 35:70. 
OLIVEIRA FILHO, A. M., 1988. Development of insecticide formulations and determination of dosages and application schedules to fit specific situations. Revista Argentina de Microbiología, 20:39-48.

OLIVEIRA FILHO, A. M., 1989. Cost-efectiveness analysis in Chagas' disease vectors control interventions. Memórias do Instituto Oswaldo Cruz, 84:409-417.

OLIVEIRA FILHO, A. M., 1994. Techniques for the entomological evaluation of the control of Chagas' disease vectors. Acta Toxicológica Argentina, 2:5255.

OLIVEIRA FILHO, A. M., 1995. Recent advances on the use of slow-release insecticide formulations against triatomines. Revista da Sociedade Brasileira de Medicina Tropical, 28:74-78.

OLIVEIRA FILHO, A. M., 1996. Review and Recommendations of Evaluation Methods and Criteria for Testing Insecticides against Triatomines. WHO Document No. CTD/WHOPES/IC/WP.4.4.1. Geneva: World Health Organization.

OLIVEIRA FILHO, A. M., 1997. Uso de nuevas herramientas para el control de triatominos en diferentes situaciones entomológicas en el continente americano. Revista da Sociedade Brasileira de Medicina Tropical, 30:41-46.

OLIVEIRA FILHO, A. M., 1998. The contribution of vector control campaigns to the insecticides environmental pollution in Brazil. In: Fifth International HCH and Pesticides Forum, Abstracts, pp. 65-71, Bilbao: IHOBE - Sociedad Pública de Gestión Ambiental.

OLIVEIRA FILHO, A. M. \& MELO, M. T. V., 1987. Field trial of the pyrethroid bifenthrin (OMS 3024) against triatomines in an area of T. infestans colonization - Riachão das Neves, BA. Ciência e Cultura, 39:817.
OLIVEIRA FILHO, A. M.; MELO, M. T. V.; SANTOS, C. E.; FIGUEIREDO, M. J. \& SILVA, E. L., 1988a. Comparative activity of the pyrethroids esfenvalerate, cyphenothrin, prallethrin, pynamin forte and neo-pynamin forte by topical application of $T$. infestans. In: V Reunião de Pesquisa Aplicada em Doença de Chagas, Resumos, p. 89, Araxá: Superintendência de Campanhas de Saúde Pública, Ministério da Saúde.

OLIVEIRA FILHO, A. M.; SANTOS, C. E. \& MELO, M. T. V., 1988b. Field trials for the determination of dose of cyfluthrin (OMS 2012) to be recommended for the control of triatomines. In: V Reunião de Pesquisa Aplicada em Doença de Chagas, Resumos, p. 93, Araxá: Superintendência de Campanhas de Saúde Pública, Ministério da Saúde.

PINCHIN, R.; OLIVEIRA FILHO, A. M. \& GILBERT, B., 1979. Field trial of slow-release formulations of chlorpyrifos and malathion for the control of Chagas' disease vectors. Revista Brasileira de Malariologia e Doenças Tropicais; 31:19-27.

PINCHIN, R.; OLIVEIRA FILHO, A. M.; FANARA, D. M. \& GILBERT, B., 1980. A preliminary field trial of decamethrin (OMS 1998) for the control of Triatoma infestans. WHO Document No. VBC/80.797. Geneva: World Health Organization.

PINCHIN, R.; OLIVEIRA FILHO, A. M. \& GILBERT, B., 1981a. Field trial of Permethrin for the control of Triatoma infestans. Bulletin of the Pan American Health Organization, 15:370-376.

PINCHIN, R.; FANARA, D. M.; CASTLETON, C. W. \& OLIVEIRA FILHO, A. M., 1981b. Comparison of techniques for detection of domestic infestations with Triatoma infestans in Brazil. Transactions of the Royal Society of Tropical Medicine and Hygiene, 75:691-695.

PINCHIN, R.; FANARA, D. M. \& OLIVEIRA FILHO, A. M., 1984. A village scale field trial of Bendiocarb (OMS 1394) for the control of the Chagas' disease vector Triatoma infestans in Brazil. Insect Science and its Application, 5:121-126. 